\title{
High-frequency ultrasound associated with dermoscopy in pre-operative evaluation of basal cell carcinoma*
}

\author{
Elisa de Oliveira Barcaui ${ }^{1}$ \\ Paulo Marcos N. Valiante ${ }^{1}$
}

\author{
Antonio Carlos Pires Carvalho ${ }^{1}$ \\ Carlos Baptista Barcaui ${ }^{2}$
}

DOI: http://dx.doi.org/10.1590/abd1806-4841.20143176

\begin{abstract}
The recent development of high-frequency ultrasound, associated with the improved sensitivity in color Doppler, enabled the identification of various skin structures and layers. In basal cell carcinoma, the 22 $\mathrm{MHz}$ frequency ultrasound permits the delimitation of tumor margins, while color Doppler, determines its vascularization. We present two cases in which the association of both exams allowed an in vivo analysis of the tumor's morphology, size, thickness and vascularization, thus contributing to a better pre-operative evaluation.

Keywords: Carcinoma, basal cell; Dermoscopy; Ultrasound
\end{abstract}

\section{BACKGROUND}

Used since the 70s in dermatology, ultrasonography is based on the reflection of sound waves throughout the tissues. ${ }^{1,2}$ According to the anatomical structure, its vascularization and density, the ultrasound waves are reflected back to the transducer that converts them into a gray scale, observed on the monitor. ${ }^{3}$ The higher the frequency of the waves emitted by the transducer, the better the spatial resolution and subsequent visualization of structures near it. The introduction of transducers with frequency higher than $15 \mathrm{MHz}$ produced the high-frequency ultrasound (HFUS). The shortest wavelength obtained by this frequency allowed a better assessment of superficial structures, significantly expanding its use in cutaneous diseases. ${ }^{4}$

In normal skin, the echogenicity of each layer depends on its main component, which in the epidermis is represented by keratin, in the dermis by collagen and in the subcutaneous tissue by fat lobules. In the ultrasound image, the epidermis appears as a hyperechoic line, the dermis as a hyperechoic band less bright than the epidermis and the subcutaneous tissue as a hypoechoic layer with hyperechoic fibrous septa in between. ${ }^{3}$
Dermoscopy is a complementary exam of great impact in dermatological practice that permits an early differentiation between malignant and benign cutaneous lesions. A study by Altamura et al, aimed at determining the accuracy of this method in the diagnosis of basal cell carcinoma, demonstrated a high sensitivity rate $(87 \%){ }^{5}$

Basal Cell Carcinoma (BCC) is an epithelial neoplasm, which corresponds to approximately 75$80 \%$ of cutaneous tumors in middle-aged individuals with fair skin. It usually affects areas exposed to solar radiation, with the highest incidence rate in the face. ${ }^{6}$ Overestimation of the tumor area can lead to unnecessary aesthetic problems. On the other hand, incomplete excisions are charged with changing the tumor structure, thus generating a more aggressive behavior. $^{7,8}$ Sartore et al reports that 5 to $50 \%$ of BCC are incompletely excised. ${ }^{7,9}$

The determination of the tumor extent and the adequate safety margins are of paramount importance for surgical intervention. ${ }^{9}$ With dermoscopy, it is possible to assess the extension of the lesion in the longitudinal and horizontal axes. However, it is not feasible to determine its depth and the potential invasion of adjacent structures, such as cartilage and muscle, based only

Received on 28.09.2013.

Approved by the Advisory Board and accepted for publication on 04.11.2013.

* Work performed at the Radiology and Pathology Department at Faculdade de Medicina da Universidade Federal do Rio de Janeiro (FM-UFRJ) - Rio de Janeiro (RJ), Brazil.

Conflict of interest: None

Financial funding: None

Universidade Federal do Rio de Janeiro (UFRJ) - Rio de Janeiro (RJ), Brazil.

Universidade do Estado do Rio de Janeiro (UERJ) - Rio de Janeiro (RJ), Brasil. 
on clinical and dermoscopic evaluation. With HFUS, it is possible to delimit the tumoral margin based on the difference in refraction between the hypoechoic tumor area and the hyperechoic perilesional region. In parallel, it is possible to assess tumor vascularization, its nature and distribution with color Doppler exams.

\section{CASES REPORT}

Two patients, one male and one female, aged 67 and 73 years presented lesions on the nose and right flank, respectively (Figures 1 and 2). Dermoscopy (DermLite DL3, 3rd Gen, USA) and 22MHz HFUS (Esaote, My Lab Touch, Italy) were performed in both patients. The male patient reported a past medical history of having one lesion removed in the same location, three years ago. Dermoscopic examination showed, in both cases, the presence of arborizing telangiectasias and ovoid nests (Figures 3 and 4). HFUS (22 MHz) demonstrated a hypoechoic lesion in the dermis of the first patient, measuring $1 \mathrm{~mm}$ deep by $1.9 \mathrm{~mm}$ in its largest diameter, surrounded by a slightly less echogenic area consistent with fibrosis (Figure 5A). Color Doppler exam showed the presence of blood vessels permeating the tumor (Figure 5B). Hypoechoic lesions measuring $1 \times 3.5 \mathrm{~mm}$, delimitated by the hyperechoic surrounding dermis were observed on the second patient; color Doppler aspects were similar to those of the previous exam (Figure 6A and $6 \mathrm{~B})$. Patients underwent excision of the lesions after adequate delineation of tumoral margins, guided

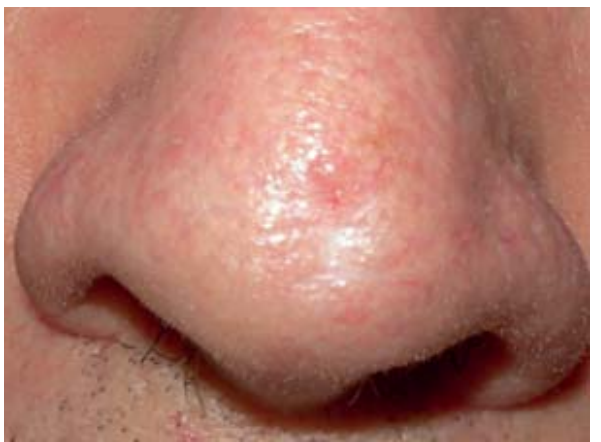

FIGURE 1:

Normochrom ic papular lesion, on the nose, over prior surgical scar

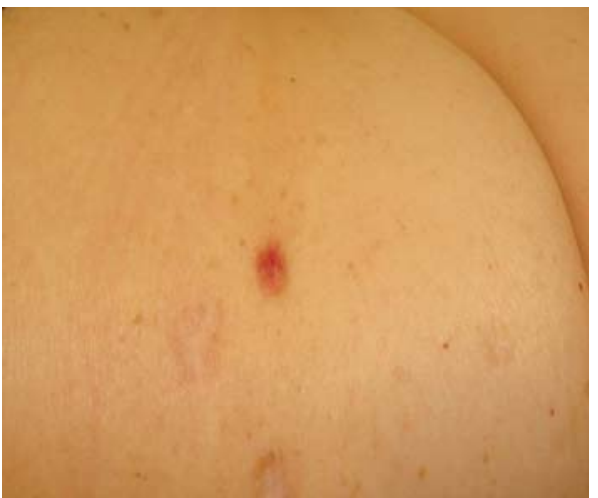

FIGURE 2: Erythematou $\mathrm{s}$ papule on the right flank

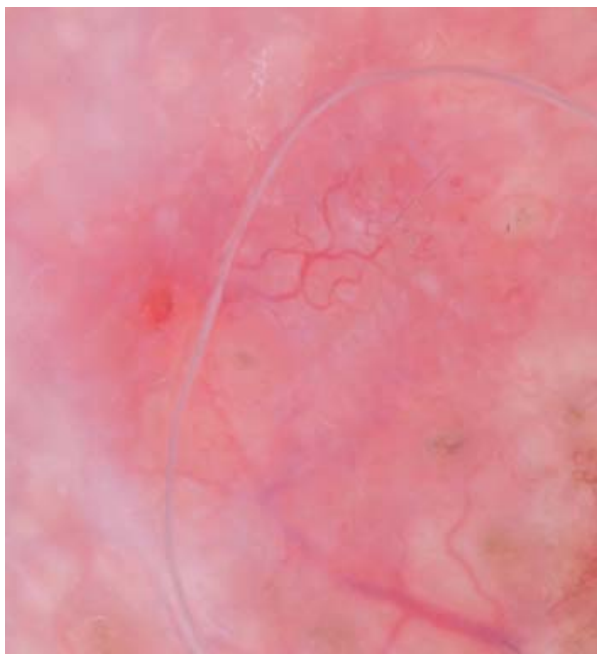

FIGURE 3:

\section{Dermoscopy}

showing classic arborizing telangiectasias and slightly pig$\mathrm{m}$ e $\mathrm{nt}$ e $\mathrm{d}$ amorphous area

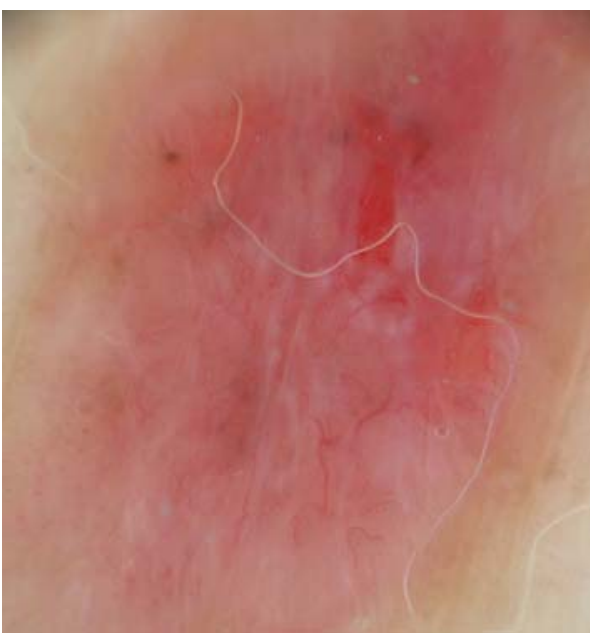

Figure 4:

Dermoscopy presenting white-reddish glossy areas and fine telangiectasias. Dis crete ovoid nests

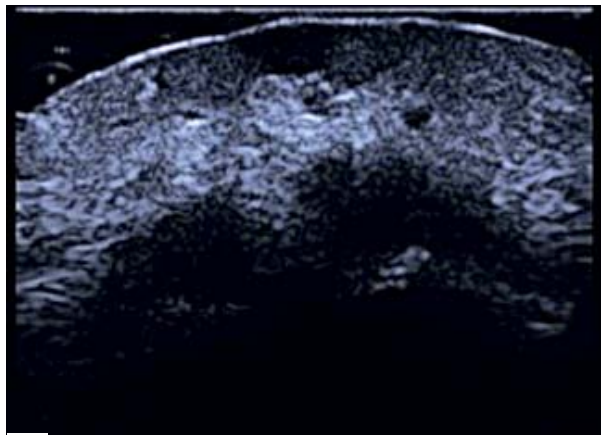

Figure 5:

A. Gray scale in the ultrasound shows well delimited, hypoechoic oval lesion, involving the dermis. B. Color D o p p l e $r$ shows increased vascularity on the tumor 

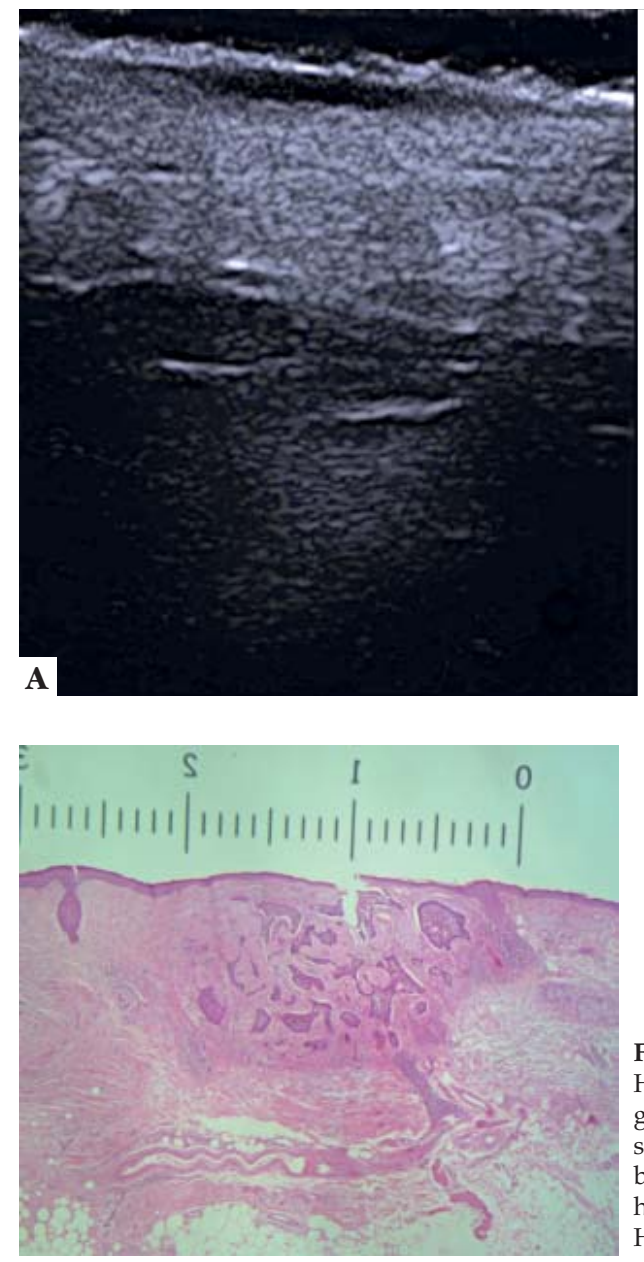

FigURE 7: Histopatholo gical exam shows a basaloid epithelial tumor. $H \& E 10 X$

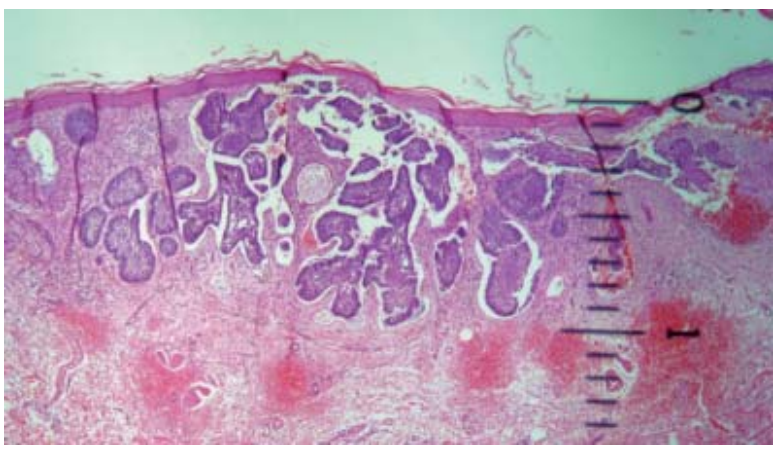

FIGURE 8: Histopathological exam: basaloid lesion infiltrating the dermis. H\&E, 40X

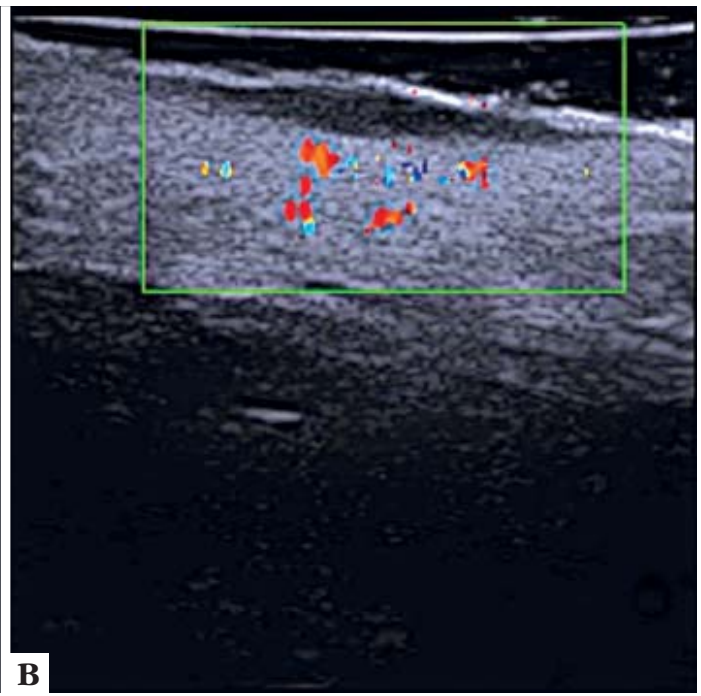

Figure 6:

A. $22 \mathrm{MHz}$ HFUS showing hypoechoic tumoral lesion. B. Color Doppler shows blood vessels positioned in the inferior portion of the lesion

by dermoscopy and HFUS. Histopathological examination revealed, in the first case, nests of basaloid cells amidst old scarring, reaching $1 \mathrm{~mm}$ deep and $1.8 \mathrm{~mm}$ laterally (Figure 7). The second patient had a similar histopathological result, with the lesion measuring 1 $\mathrm{mm}$ deep and $3.5 \mathrm{~mm}$ laterally (Figure 8).

\section{DISCUSSION}

Currently, histopathological examination is the gold standard for diagnosis and morphological and structural assessment of BCC. However, new techniques for in vivo investigation have been used to expedite diagnosis and optimize pre-operative evaluation.

Studies indicate that HFUS represents an innovative method for exploring cutaneous tumors, including BCC. Unfit to assess tumor cellularity, this exam cannot be used to confirm diagnosis, but it enables a detailed preoperative study: by assessing the different skin layers and their respective thicknesses, the tumor size and involvement of deep planes. Color Doppler exam can estimate the blood flow on the lesion and its surroundings.

Histopathological analysis confirmed the assessment of pre-operative tests.

In summary, we report two cases that exemplify how the association of HFUS with dermoscopy adds substantial value to the analysis of tumoral dimensions, which also permits the safe determination of their margins and vascularization patterns prior to tumor excision.] 


\section{REFERENCES}

1. Wortsman $\mathrm{X}$, Wortsman J. Clinical usefulness of variable-frequency ultrasound in localized lesions of the skin. J Am Acad Dermatol. 2010;62:247-56.

2. Kleinerman R, Whang TB, Bard RL, Marmur ES. Ultrasound in dermatology: principles and applications. J Am Acad Dermatol. 2012;67:478-87.

3. Wortsman X. Common Applications of Dermatologic Sonography. J Ultrasound Med. 2012;31:97-111.

4. Crisan M, Crisan D, Sannino G, Lupsor M, Badea R, Amzica F. Ultrasonographic staging of cutaneous malignant tumors: an ultrasonographic depth index. Arch Dermatol Res. 2013;305:305-13.

5. Altamura D, Menzies SW, Argenziano G, Zalaudek I, Soyer HP, Sera F, et al. Dermatoscopy of basal cell carcinoma: Morphologic variability of global and local features and accuracy of diagnosis. J Am Acad Dermatol. 2010;62:67-75

6. Andrade P, Brites MM, Vieira R, Mariano A, Reis JP, Tellechea 0, et al. Epidemiology of basal cell carcinoma and squamous cell carcinoma in a department of dermatology: a 5 year review. An Bras Dermatol. 2012;87:212-9.

7. Wortsman X. Sonography of Facial Cutaneous Basal Cell Carcinoma. A First Line Imaging Technique. J Ultrasound Med. 2013;32:567-72.

8. Ocanha JP, Dias JT, Miot HA, Stolf HO, Marques ME, Abbade LP. Relapses and recurrences of basal cell face carcinomas. An Bras Dermatol. 2011;86:386-8.

9. Nassiri-Kashani M, Sadr B, Fanian F, Kamyab K, Noormohammadpour P, Shahshahani MM, et al. Pre-operative assessment of basal cell carcinoma dimensions using high frequency ultrasonography and its correlation with histopathology. Skin Res Technol. 2013;19:e132-8.

\author{
MAILING ADDRESS: \\ Elisa de Oliveira Barcaui \\ Rua Rodolpho Paulo Rocco, 255 - Cidade Universitária \\ 21941-913 - Ilha do Fundão - RJ \\ Brazil \\ E-mail: ebarcaui@gmail.com
}

How to cite this article: Barcaui EO, Carvalho ACP, Valiante PMN, Barcaui CB. High-frequency ultrasound associated with dermoscopy in pre-operative evaluation of basal cell carcinoma. An Bras Dermatol. 2014;89(5):828-31. 